\title{
Biopsy follow-up in patients with isolated atypical small acinar proliferation (ASAP) in prostate biopsy
}

\author{
Luca Leone $^{1}$, Vito Lacetera ${ }^{1}$, Rodolfo Montironi ${ }^{2}$, Ubaldo Cantoro ${ }^{1}$, Alessandro Conti ${ }^{1}$, \\ Giulia Sbrollini ${ }^{1}$, Luigi Quaresima ${ }^{1}$, Luciana Mariani ${ }^{1}$, Giovanni Muzzonigro ${ }^{1}$, Andrea Benedetto Galosi ${ }^{3}$ \\ ${ }^{1}$ Institute of Urology, ${ }^{2}$ Institute of Pathology, Azienda Ospedaliera-Universitaria, Polytechnic University of Marche Region, \\ Ospedali Riuniti Ancona, Italy; \\ ${ }^{3}$ Division of Urology, "Augusto Murri" General Hospital, ASUR Marche, Fermo, Italy
}

\begin{abstract}
Summary The incidence of prostate cancer (PCA) was evaluated in 155 patients with isolated Atypical Small Acinar Proliferation (ASAP) found on initial prostate biopsy, after a medium-term follow-up (40 months) with at least one re-biopsy. Clinical and histological data were analysed. Cancer was detected in 81 of 155 (52.3\%). The cancer detection rate was $71.6 \%, 91.3 \%$, $97.5 \%, 100 \%$ at the $1^{\text {st }}$ re-biopsy, $2^{\text {nd }}, 3^{\text {rd }}$, and $4^{\text {th }} r e$ biopsy respectively. At the uni- and multivariate analyses, prostate volume $(\leq 30 \mathrm{cc})$, transition zone volume $(\leq 10$ $c c)$, small core length at the initial biopsy $(\leq 10 \mathrm{~mm})$ and few number of cores at initial biopsy $(\leq 8)$ are predictive of cancer. Furthermore, tumour characteristics on the whole surgical specimens was assessed in 30 men: 13 of $30(43 \%)$ had clinically relevant cancer (volume $>0.5 \mathrm{ml}$ or/and Gleason score $\geq 7$, or pT3). Most of relevant cancers were detected in the distal apex, anterior gland and midline. These anatomical sites could be under-sampled at the initial biopsy using the transrectal approach.

Our data suggest that follow-up biopsy is recommended in all cases of isolated ASAP detected after biopsy using endfire transrectal probe. The re-biopsy strategy should increase the number of cores (or a saturation biopsy), focusing on area of ASAP in the initial biopsy, but also including the under-sampled areas (anterior gland, distal apex and midline) to detect clinically relevant cancers.
\end{abstract}

KEY WORDS: Atypical small acinar proliferation; Prostate neoplasms; Biopsy; Diagnosis; Radical prostatectomy.

Submitted 3 October 2014; Accepted 31 October 2014

\section{INTRODUCTION}

The Atypical Small Acinar Proliferation (ASAP) is a pathological definition and is not a disease (1). ASAP is a very small area composed by few glans with atypical epithelium suspect, but non-diagnostic, for cancer and therefore it is not considered a disease such a pre-neoplastic lesion as high grade Prostatic Intraepithelial Neoplasia (PIN) or cancer (2). ASAP can be isolated or associated to concomitant high grade PIN or associated to adenocarcinoma (Figure 1). We evaluated the isolated ASAP only, and cases associated with PIN or cancer were excluded. ASAP isolated is found in about the $5 \%$ of prostate biopsy in men with long life expectancy (> 20 years) (1-3). Thus isolated ASAP in biopsy is an important clinical dilemma for patients and for physicians in order to identify concurrent relevant cancer. We evaluate retrospectively our experience at medium-term follow-up. Principal aim of our study was to evaluate the prostate cancer after 40 months of follow-up with at least 1 re-biopsy. Secondary aims was to describe tumour characteristics and anatomical location analysing whole surgical specimens in men who underwent surgery for cancer at repeated biopsy.

\section{MATERIAL AND METHOdS}

From April 1998 to February 2014, we evaluated 4862 biopsies performed in our Hospitals using transrectal echo-guided mapping. We retrospectively selected 190 patients with isolated ASAP on biopsy. Only men who had all clinical data available including at least 1 followup re-biopsy were included in the series.

A database including histological (site, side, number and length of cores) and clinical findings (age, total and free PSA, digital rectal examination and trans-rectal ultrasound findings, prostatic volume) was analysed and matched with follow-up data. Selection criteria were: isolated ASAP, available clinical data, central review of all slides, at least 6 months follow-up with at least one repeated biopsy. Indications to re-biopsy and follow-up strategies were discussed on individual basis according to patient and urologist preference depending on patient age, life expectancy, serum PSA values, imaging (transrectal ultrasound or magnetic resonance) and digital rectal exploration findings. The technique of transrectal biopsy was previously described and included the use of end-fire ultrasound probe and sandwich technique to pre-embedding the biopsy fragment between 2 sponges (4). No more than two cores per cassette were collected in order to avoid tissue loss during micro-section.

Follow-up time was considered from the initial biopsy with ASAP to the last follow-up visit or date of cancer diagnosis. Surgery was proposed in patients with cancer on re-biopsy and candidate to surgery according to 
European guidelines. Whole prostatectomy specimens were analysed by a step section protocol and cancer volume was calculated. Central review of all slides (including initial and follow-up biopsies) and surgical specimens was made by a single expert uro-pathologist (5). Before diagnosis of isolated ASAP, 28 of 155 patients (18\%) had previous negative biopsy. All data were analysed using univariate and multivariate analyses to detect clinical factors associate with cancer or not.

Confidence interval was calculated at 95\% and standard deviation $( \pm)$ value was reported.

\section{RESULTS}

Isolated ASAP was identified in 3.9\% of patients (190/4862). 155 of 190 ASAP were selected and analysed, 40 patients were excluded because lost at follow-up or declined re-biopsy.

The mean follow-up was 40.2 months (min 6, max 143). Baseline characteristics of the 155 patients with isolated ASAP, at the original biopsy, were the following: median number of cores at the initial biopsy was 14.5 cores (range 6 to 24$)$; median age was 65.1 years $( \pm 7.2$, C.I. 52-77); median PSA was $6.9 \mathrm{ng} / \mathrm{ml}$ ( \pm 4.8, C.I. 2-16.3); mean prostate volume was $47 \pm 23 \mathrm{cc}$.

Cancer was detected overall in 81/155 (52,3\%). 114 patients had the $1^{\text {st }}$ re-biopsy, 29 had the $2^{\text {nd }}$ re-biopsy, 7 had the $3^{\text {rd }}$ re-biopsy, 3 the $4^{\text {th }}, 1$ had the $5^{\text {th }}$ and 1 had the $6^{\text {th }}$ re-biopsy. Specific cancer detection rate in each follow-up re-biopsy set was: $71.6 \%(58 / 81)$ at the $1^{\text {st }}$ rebiopsy, $19.7 \%(16 / 81)$ at $2^{\text {nd }}$, but declined to $6.1 \%$ $(5 / 81)$ at the $3^{\text {rd }}$ re-biopsy, and $2.4 \%(2 / 81)$ at $4^{\text {th }}$ rebiopsy. No cancer was found at the 5 th and 6 th re-biopsy $(0 / 81)$ (Table 1$)$

Therefore the cancer detection rate was $71.6 \%, 91.3 \%$, $97.5 \%, 100 \%$ at the 1 st re-biopsy, $2^{\text {nd }}, 3^{\text {rd }}$, and $4^{\text {th }}$ rebiopsy after ASAP diagnosis. The median number of cores was 15.6 ( $\min 6, \max 59$ cores, \pm 6.7 ) at the 1 st rebiopsy and 13.7 ( $\min 8-\max 24$ cores, \pm 4 ) at the $2^{\text {nd }}$ re-biopsy. The number of cores was decided according to the volume of the prostate gland. Only few variables had a significantly positive association with prostate cancer in univariate and multivariate analyses: total prostate volume $(\leq 30 \mathrm{cc}$ ) and transitional zone volume ( $\leq 10 \mathrm{cc}$ ), PSA velocity, small core length at the initial biopsy $(\leq 10$ $\mathrm{mm})$ and few number of cores at initial biopsy $(\leq 8)$ are predictive of cancer in follow-up. Pathological analysis of the whole prostate was available in 30 of 81 patients who underwent retropubic or robotic radical prostatectomy. Pathology report after radical surgery detected 17 insignificant cancer (liberal definition: T2, Gleason Score $3+3=6$, volume $<0.5 \mathrm{ml}$ ) and 13 relevant cancer (volume $>0.5 \mathrm{ml}$, presence of Gleason pattern 4 or/and stage $\geq \mathrm{T} 3 \mathrm{a})$.

Relevant disease was detected in 43\% (13/30) cases. Extra-capsular disease or seminal vesicles invasion (pT3 $\mathrm{a}-\mathrm{b}$ ) was found in 8 cases, positive margin ( $\mathrm{pR} 1$ ) was found in 3 cases, association of both findings was detect- ed in 1 case. Significant disease was observed also in 5 patients who had a low volume of cancer $(<0.5 \mathrm{ml})$ in the anterior gland (subcapsular and fibromuscolar anterior stroma), distal apex, midline area in the peripheral gland (sub-urethral) (Figures 2, 3).

\section{Discussion}

In our experience the cancer detection rate is $52.3 \%$ while in literature the average incidence is $40.7 \%$ (1760\%) (6-10): our series also includes a long follow-up (40 months) and repeated biopsy (range 1 to 6). We found most cancer $(71.6 \%)$ within the first re-biopsy, as reported in literature (7-9).

Usually we found large tumours frequently located in the anterior zone of large prostate. In our experience, if clinical findings are positive (nodules at the palpation or/and ultrasound), histological inflammation is not present, the initial biopsy is poor in quality $(<9$ cores, mean core length $<14 \mathrm{~mm}$ ), the cancer in follow-up biopsy raise to 70\% (Figure 1: left side).

There are few series about the cancer pathology based on prostatectomy after initial finding of ASAP (10-11). Based on previous experiences, cancer can be clinically relevant or not relevant: the first case is a massive tumour

\section{Figure 1.}

pT2 cancer is under-sampled by transrectal biopsy: in the right side a low grade and small volume cancer is missed and tangentially sampled (2 arrows); in the left side, a large volume cancer is near-missed (arrow).

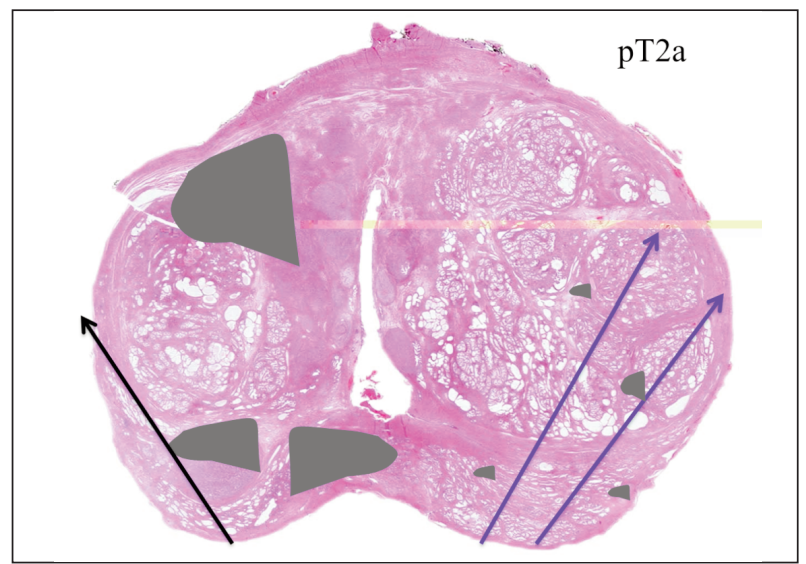


Figure 2.

A: axial image of the distal apex, using end-fire transrectal probe.

C: biopsy in the anterior stroma of the transition zone (arrow)

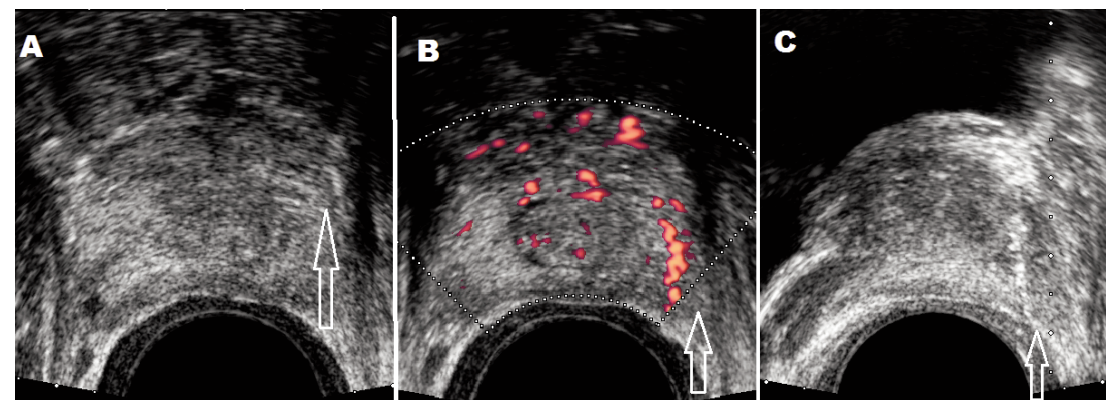

Figure 3.

Hypoechoic area in the anterior gland, observed with end-fire probe (arrow), A: eco-power-doppler.

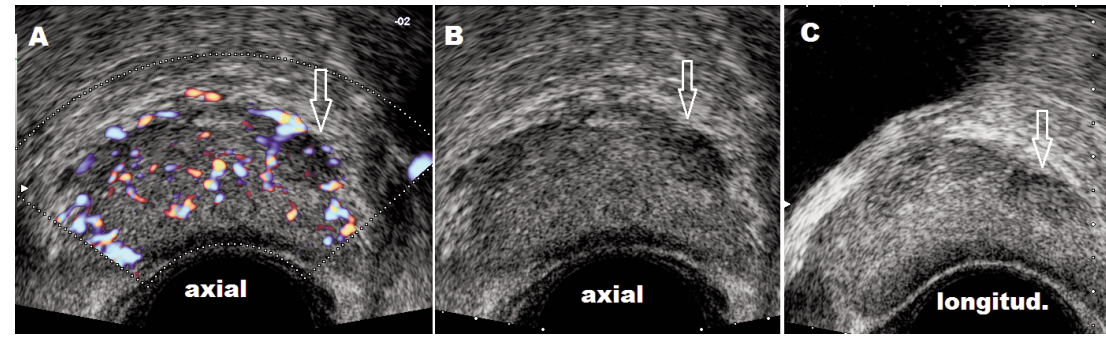
Arrow show biopsy track. B: ecopower-doppler.

cancer leading to misinterpretation of few atypical glands. We support this hypothesis if clinical findings and imaging are negative, inflammation is prominent in biopsy specimen, the initial biopsy is of good quality ( $>12$ cores, mean core length $\geq 14 \mathrm{~mm}$ ); in this group, the cancer rate is low (25\%) in follow-up biopsy (data not shown).

\section{Conclusions}

In patients with isolated ASAP, the overall cancer detection rate is 52.3\% after 40 months of follow-up and at least 1 repeated biopsy. Most cancer (97.6\%) are detected with the 3rd repeated biopsy. Cancer detected after initial ASAP is still curable, however 8 of $30(26.6 \%)$ cases submitted to surgery had adverse pathological features and $13 / 30(43 \%)$ are clinically relevant. Five relevant tumours had low volume associated with particular anatomical location (anterior, subcapsular o suburethral).

The re-biopsy strategy should increase the number of cores, or a saturation biopsy, focusing on the area of ASAP in the initial biopsy, but also including tangentially sampled because localized near the biopsy track $(55 \%)$, the second one is a microfocus $(<0.5 \mathrm{cc}$ GS $3+3)(45 \%)$.

Cancer biopsy under-sampling is a convincing option in relation to uncommon location or small volume of cancer or both (Figure 1) (11-13). The under-sampling of small volume cancer can be divided in two categories: low risk (well differentiated, Gleason score 3 +3 , T2) or insignificant, and high risk (Gleason score $>6$ or T3) or significant cancer. In our experience high risk and small volume cancer was present in 5 cases.

Therefore not all small volume cancers are insignificant cancer. This observation is important for diagnostic imaging (ultrasound or magnetic resonance), where threshold volume for detection is $0.5 \mathrm{cc}$. Furthermore, the anatomical location of tumour foci could be not usual as generally believed: anterior gland (subcapsular and fibromuscolar anterior stroma), distal apex, midline area (sub-urethral) of the peripheral zone may harbour cancer. Unusual anatomical location associated with low cancer volume may explain because biopsy under-sampling occurs. In fact biopsy shows only a small foci of ASAP on initial biopsy.

Therefore a different strategy in the repeated biopsy should include the anterior area (Figure 2) and midline zone. This strategy, that we adopted resulted in diagnosis and treatment of relevant cancer even of small volume cancers, as we observed in five cases. Our detection of ASAP (3.9\% of prostatic biopsies) is similar to literature data (5\%) (6-8), defining good quality control of our experience. Also inflammation could mimic ASAP or the anterior gland, distal apex and midline that could not be sampled initially.

\section{REFERENCES}

1. Montironi R, Scattoni V, Mazzucchelli R, et al. Atypical Foci Souspicious but not diagnostic of malignancy in prostate needle biopsies (also referred to as "atypical small acinar proliferation suspicious for but not diagnostic of malignancy"). Eur Urol. 2006; 50:666-674.

2. Epstein JI, Herawi M. Prostate needle biopsies containing prostatic intraepithelial neoplasia or atypical foci suspicious for carcinoma: implications for patient care. J Urol. 2006; 175:820-834.

3. Epstein JI, Yang XJ. "Finding of atypical glands suspicious for canIL: Lippincott Williams \& Wilkins. 2002; 177-184.

4. Galosi AB, Lacetera V, Leone L, et al. Biopsy follow-up in patients with isolated atypical small acinar proliferation (ASAP) on prostate needle biopsy. Anticancer Research. 2011; 31: 1938-1939.

5. Galosi AB, Lacetera V, Cantoro D, et al. Carcinomi prostatici di piccolo volume $(<0,5 \mathrm{ml})$ : caratterisctiche e implicazioni cliniche. Urologia 2009; 76:236-241.

6. Schlesinger C, Bostwick DG, Iczkowski KA. High grade prostatic intraepithelial neoplasia and atypical small acinar proliferation: predictive value for cancer in current practice. Am J Surg Pathol. 2005; 29:1201-1207.

7. Postma R, Roobol M, Schroder FH, et al. Lesions predictive for prostate cancer in a screened population: first and second screening round findingd. Prostate. 2004; 260-266.

8. Chan TY, Epstein JI. Follow-up of atypical prostate needle biopsies suspicious for cancer. Urology. 1999; 53:531-535. cer", in: Epstein JI, Yang XJ: Prostate Biopsy Interpretation. Chicago, 
9. Iczkowski KA, Chen HM, Yang XJ, et al. Prostate cancer diagnosed after initial biopsy with atypical small acinar proliferation suspicious for malignancy is similar to cancer found on initial biopsy. Urology. 2002;60:851.

10. Kopp RP, Parsons JK, Shiau J, et al. Prostate atypia: clinical and pathological variables associated with cancer diagnosis on repeat biopsy. Prostate Cancer Prostatic Diseases. 2011; 14:149-154.

11. Chen YB, Pierorazio PM, Epstein JI. Initial atypical diagnosis with carcinoma on subsequent prostate needle biopsy: findings at radical prostatectomy. J Urol. 2010; 184:1953-1957.

12. Galosi AB, Montironi $R$, Mazzucchelli R, Muzzonigro G. Clinical pathology of prostate cancer: focus on diagnosis, predictive and prognostic factors and quality indicators. Arch Ital Urol Androl. 2007; 79:45-51.

13. Galosi AB. ASAP nella biopsia prostatica: gestione nella pratica clinica. Urotime. 2011; 34:11-12.

\section{Correspondence}

Luca Leone, MD, Resident in Urology

lucaleone85@virgilio.it

Vito Lacetera, MD, FEBU, Urologist

vlacetera@gmail.com

Ubaldo Cantoro, MD, Resident in Urology

ubaldocantoro@tiscali.it

Alessandro Conti, MD, Urologist

alessandroconti@hotmail.com

Giulia Sbrollini, MD, Resident in Urology

giuliasbrollini@libero.it

Luigi Quaresima, Resident in Urology

luigiquaresima@yahoo.it

Luciana Mariani, MD, Urologist

lucianamariani@virgilio.it

Giovanni Muzzonigro, MD, Director, Professor of Urology

g.muzzonigro@univpm.it

Institute of Urology, Azienda Ospedaliera-Universitaria, Polytechnic

University of Marche Region, Ospedali Riuniti Ancona, Italy

Rodolfo Montironi, MD, FRCP, Professor of Pathology

r.montironi@univpm.it

Institite of Pathology, Azienda Ospedaliera-Universitaria, Polytechnic

University of Marche Region, Ospedali Riuniti Ancona, Italy

Andrea Benedetto Galosi, MD, PhD, Urologist (Corresponding Author)

galosiab@yahoo.it

Division of Urology, Dept. of Surgery, "Augusto Murri" General Hospital, Area Vasta 4, Az. Sanitaria Unica Regione Marche

63900 Fermo (FM), Italy 